\title{
ANN based LFC with Coordination strategies of DERs in Hybrid Isolated Micro-Grid Environment.
}

\author{
Srikanth Boyini ${ }^{1}$, Srividya Devi Palakaluri ${ }^{2,}{ }^{*}$, and Rekha Mudundi $^{3}$ \\ ${ }^{1}$ Research Scholar, EE Department, GRIET, Hyderabad, Telangana \\ ${ }^{2}$ Associate Professor, EE Department, GRIET, Hyderabad, Telangana \\ ${ }^{3}$ Assistant Professor, EE Department, GRIET, Hyderabad, Telangana
}

\begin{abstract}
This paper provides a load frequency control (LFC) of a micro grid with renewable energy resources (RES). The operation of micro grid with a low inertia system leds to disturbances in power system. The disturbances in frequency is more in micro grid than conventional power system. So there should be a fast recovery of changes in frequency with existing system and interconnected system (RES). Active power injection is the main scheme to control frequency of a system. The matlab simulink tells us that different active power injection system contribute for the fast control of grid frequency with PID controller. The use of ANN technology to this system the load frequency control can be illustrated in faster rate of its recovery. An ANN controller is investigated which handles the inputs collectively in each sector of the power system. Back-transmission time is normally used in the study for neural network education. The performance of the power system is simulated independently with a typically integrated conventional controller and ANN controller. A complete spectrum of small signals is introduced for RESs in the isolated microgrid and a correct role in frequency control studies is taken into account.
\end{abstract}

\section{Introduction}

The prerequisite is to improve the confidence, economics and environment for conventional systems to introduce micro-grid (MG) into the energy system[1,2]. It seems obvious that the $\mathrm{MG}$ is a power storage device that includes everything. RES generators are commonly dispersed and employed in MGs[3,4] for load controlled and storage instruments (DGUs). RES is usually spaced out. The distributed generations typically included in the MG[5] studies are wind energy generators, photovoltaic (PV) panels, fuel (FCs), diesel (DEG) and battery storage equipment for flywheels (BESSs).The successful use of the DG units satisfies detrimental environmental constraints not only in order to achieve the spread of electricity consumption. Although the MG Framework for DGUs offers a lot of advantages, there are a number of issues associated with the monitoring, stabilisation, and efficiency of the MGs in terms of a greater variety of power sources with a wide range of dynamics[6]. The MG connection mode could in some way exacerbate the problems indicated. In two different ways the MGs work: (1) Isolated mode, and (2) Grid link mode[7], as prior study shows.

Unlike the RES structure in the island mode, a rendered-connecting mode involves intermittent change in RESs using smart, flexible AC MG management strategies to ensure robust performance and stability by remedying a connected power grid which reduces the disturbances in charge compared to the island mode. The SACP system in an island state has major obstacles and needs to be assessed for several reasons. The main generation of AC-MG fleets is mainly DER.This appears to result in a high frequency change in the separate microgrid from the synchronous generator (RoCoF).

The RoCoF is more than $0.2 \mathrm{~Hz} / \mathrm{s}$ in the clearness of the Low Inertia Grid in conventional systems. Therefore, the necessity to provide suitable auxiliary services, such as inertia responses and primary frequency response, is unavoidable for all inverter-related technologies. Secondly, the provision of MG stable services does not provide the whole MG swiftly, which could lead to an aggravation of the issue. When the system fail and activates a safety system, the RoCoF in the MG reduces breakage rate to its contingency limit and is able to separate each electrical unit. Therefore, support for frequency in MG must be sufficiently swift in order to avoid the drop of frequency in low frequency conditions. Third, analysing the influence of the DERs on power storage systems. In this study, storages of non-existent DER units are employed to transform them into sendable units. However, other frequency support firms may have difficulty coordinating energy storage systems. Finally, In low- and medium-voltage distribution networks [8$10]$, the method for micro-grid management is a critical concern. In other words, there are inadequate distribution networks and unclear working conditions. DER's interaction with the production plan thereby minimises

\footnotetext{
* Corresponding author: srividyadevi.p@gmail.com
} 
energy inertia[21-25]. The generator will be produced independently of the rotary component of the generator during the past years. This loss of network inertia impacts the overall frequency behaviour, which significantly weakens the stability of power supply. In fact, the effect of interconnected RESs on the inertia of the micro-range is a key aspect of micro-ride performance. The intelligent involvement of regenerated energy resource in the frequency management system[10] is one of the most successful ways in tackling this problem. For the management of a range of working conditions in isolated mode the designer should use robust and adaptable controls[11,12]. Many literatures have proposed new regulatory techniques to ensure stability for MGs under island conditions.

Several controls were submitted to help favourably govern MG's FFCs. These included strong control ([13], smart control[14], adaptive control(15]), and prediction model monitoring (MPC[12]). Controls were also proposed. This was also proposed. In this study, the authors mainly concentrated on controller design and did not discuss the dynamic models of the energy supply provided via $\mathrm{AC}-\mathrm{MG}[16]$. Charging frequency controls are a key issue in the management and monitoring of power systems in order to supply good quality, adequate and dependable power. When changes in the fields of power systems suddenly occur due to consumer demand, the links and frequency changes occur. Thus, a change in operating points in daily cycles[1-4] does not suffice to establish fixed gain control procedures, which are based on the conventional literature control theory. A fixed controller in all operating scenarios can no longer be adapted when the working point of a power system changes continually. The techniques employed for the control of load frequencies in recent years include modem controls, particularly adaptive control schemes. For complicated adaptive control configurations, the artificial neural network controller (ANN) is used as it provides quicker controls than those of others. A reverse propagation method is employed in the ANN controller proposed[5]. The project involves the regulation of interconnected energy from energy sources like Diesel (DEG), Fuel Cell (FRC), and other renewable energy sources (WTG). Since the load varies from place to place in the power system, the field frequency will initially be changed, which is followed by a disturbance in the other places. A control system should be created that functions to set the steam input van of the unit turbine in order to recover to a constant state of the frequency following a certain change in charge. In typical control settings, load frequency control systems are recognised to be supplemented by a built-in control system. Integration gains have been achieved at a level which reduces the dynamic responsiveness of the system to a minimum overhead[17-20] between rapid transitional recovery. This style of controller is slow unfortunately. Unfortunately. This means that the power system increases the transitory recovery from load disturbances. There are numerous differential equations which can govern the non-linear model because it is the best model to describe the actual system. Return time propagation methods are recommended as the learning rule utilised to manage the constant dynamics of the system through artificial neural network configurations by the neural artificial network controller[29]. To avoid overfrequency during step load disruptions, wind variations and changes in $\mathrm{mg}$ parameters may be regarded as possible reserves of active electrical injections, such as WTG, FESS, FC and DEG.

Authors suggest in their study more complex models of energy storage than prior models (ESSs). The LFC system is a multiple coordinating technique of the DER and compares outcomes for the environment MATLAB/SIMULINK, the diesel and the fuel pellet. This flywheel energy storage device then enters and considerably mitigates the frequency difference caused by load disturbances. In this study, the first area of the power system is examined as a load increase. ANN successfully manages the power system, which repeat frequency charging controls that use temporal algorithms in various operating circumstances. The results demonstrate that the traditional controller receives excellent dynamic responses in ANN setup, based on the load frequency control system's back propagation time technique.

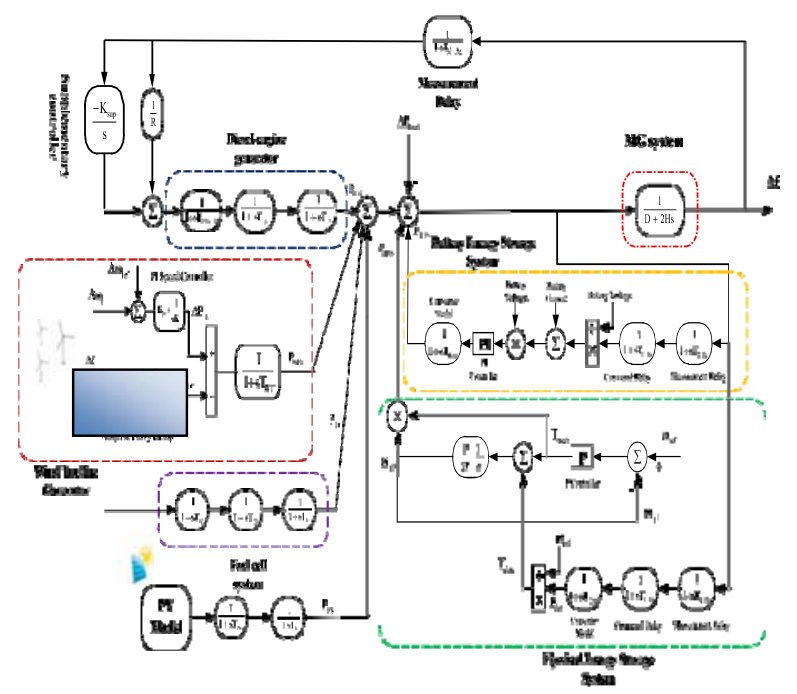

Fig. 1. Micro-grid block diagram with implemented ANN controller

\section{Modelling of micro-grid isolated DERs}

During previous investigations, there are several types of dynamic MG models available[26-28]. Most importantly, numerous authors, notably in storage units, use simpler dynamic first-order models of energy resources. This study shows that the electrical parameter associated with a normal system is a dynamic model for an isolated micro network with a little signal. In Figure 1 , power storage systems can give energy adequate for a short amount of time to protect the stability of the system. The LFC design storage time constant must also 
be taken into consideration for a few seconds. FESS and BESS is charged in time. A dynamic model of an energy storage system and a storage system based on current technological physical conditions in the next portion is described in this article (FESS). Also a rapid source of actively generating energy is the diesel generator and the fully cooperating wind turbines in the frequency control system are provided to a dynamic model in the cargo area suitable for a successful participation in the LFC system.

\section{Diesel engine generator model}

Diesel Motor Generator (DEG) is known throughout its development for its exceptionally sustainable and efficient methods of energy generation. When the demand value fluctuates, the DEG can alter the output power amount. In Figure 2 in the Frequency Control Strategy, the assigned DEG transfer function is shown. This type consists often of a controller and generator that indicate an inertia plant first-order. However, the system is included in order to highlight the inertia-wise delay to a late construction of the governor and turbine. Figure 2 shows the change in frequency $\mathrm{f} \mathrm{Tg}, \mathrm{tt}$ and the dildo De is believed to be the constant time of the governor, the time of the generator and a wise delay. Furthermore, R indicates the DEG speed control factor. For the diesel generator of the LFC type, the power increase limit block is generally regarded.

\section{Battery energy storage system (BESS) model}

Different approaches for simulating energy storage devices are available. The storage system might be considered, for instance, as a rudimentary math blackbox to generate a number of static input levels. It might be assumed that [36] the battery is still operating under normal working conditions in conjunction with BESS. A constant output pressure, while the load fluctuates with the current amount, will only be allowed to take the measuring times, control delays and the transformer delay of the model into consideration, as shown in Figure 3. The major characterization of the standard BESS area. TM De is time-constant for this model, TC De is time constant, TC is a time constant for a DC- to AC model. Moreover, the BESS paradigm is attached with a traditional PI control to reduce the electrical storage system's active power failure. This model incorporates the new term PNC that takes the entire contribution of unconventional resources into consideration. The overall power balance (Ptotal) in constant condition is the same.

$$
\Delta P_{\text {total }}=P_{D E G}+\Delta P_{N C}-\Delta \text { Pload }
$$

Model flywheel energy storage The fly-wheel energy storage device plays an important part in the correct storage and release of both microgrid functions. The microsystem, high conversion efficiency of around 80$90 \%$ and high stocking strength show the advantages of FESS in the load frequency management system. In the system of the energy storage torrent as shown in Figure 4 , the mechanical energy system is normally considered when two primary constant periods are identified: the mechanical time constant as indicated by system inertia and the electronic time constant as electronic technical elements. The authors highly encourage FESS to regulate its active and responsive strength by producing active and reactant power based on the speed of its rotating mass. in this regard. Therefore, the FESS model incorporates a speed control loop to provide an accurate and dynamic load frequency management system. Including electrical machinery, The electromechanical system's rotor acceleration equation is compared to the following system of mass:

$$
\frac{d w_{r f}}{d t}=\frac{p}{2} \frac{T_{\text {elec }}-T_{\text {mech }}}{J}
$$

The transformation of Laplace (1) is:

$$
\begin{aligned}
& s w_{r f}=\frac{p}{2 J}\left(T_{\text {elec }}(s)-T_{\text {mech }}(s)\right) \\
& w_{r f}(s)=\frac{p}{2 J} \frac{1}{s}\left(T_{\text {elec }}(s)-T_{\text {mech }}(s)\right) \\
& T_{\text {elec }}=\frac{P_{\text {elec }}}{w_{\text {ref }}} \\
& T_{\text {mech }}=K_{p}\left(w_{r f}-w_{r e f}\right)
\end{aligned}
$$

Where $\mathrm{rF}$ is the electric frequency of the machine,

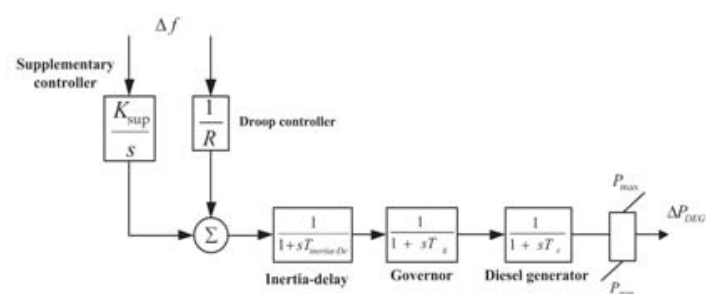

Fig. 2. Research on frequency regulation of diesel engine power frequency.

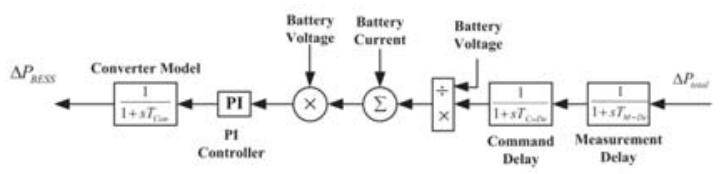

Fig. 3. Management of frequency battery power storage system. 


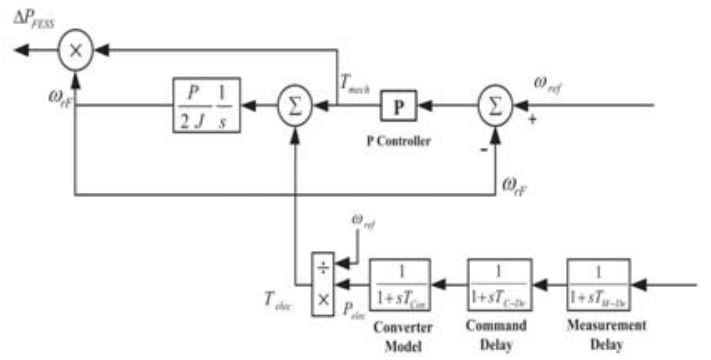

Fig. 4. The load frequency management of the flywheel energy storage device.

The number of bars, the electric torque of Teleci, the mechanical torsion at the shaft and the mass of $\mathrm{J}$ rotatory inertia are $\mathrm{kg} \mathrm{m} 2$. The electric torque is Tleci. Tleci.

\section{Wind turbine in De-loaded area model}

Therefore, the wind to wind power generator (WtG) is considered as a source of variation of the power of isolated MG and is therefore a fluctuating natural resource due of its wind to wind rate characteristics. As has been reiterated in multiple prior references in a combination of the Cp power coefficient and other physical elements, wind turbine power generation may be characterised. Overall, $\mathrm{Cp}$ is built on two fundamental components that comprise a velocity ratio and a blade spot angle. The following statement characterises the speed ratio.

$$
\lambda=\frac{R \times w_{\text {blade }}}{V_{w}}
$$

$\mathrm{R}$ is the blade velocity of the blade and radius. The link $\mathrm{Cp}$ is established by [37] and,

$c_{p}=(0.44-.0167 \beta) \times \sin \left[\frac{\Pi(\lambda-3)}{15-0.3 \beta}\right]-0.0184(\lambda-3) \beta$

In the end, the mechanical output of the wind turbine generator is specified.

$$
P_{W T}=\frac{1}{2} \rho \mathrm{A} C_{p} V_{w}{ }^{3}
$$

In this Eq. (9) the air density is $\mathrm{kg} / \mathrm{m} 3$. The area of $\mathrm{m} 2$ covering the blades is A. By shifting the working point to the right of the maximum power tracking point, the wind turbine generator can be unloaded (MPPT). The wind turbine normally works in MPPT positions constantly in Fig. 5, but Pres can inject power as a spinning reserve during load interruptions. The power output of the turbine can vary from Pmax to Pdel by adjusting the speed of its rotor between maximum and del. The maximum decompressed margin for supply of sufficient spin reserve is expected to be $15 \%$ of WTs (i.e. headroom).Additional details are provided in $[18,19,24]$ of the Strategy assumed. The de-loading factor can minimise, as has already been said, the amount of active power infusion.

$$
\begin{aligned}
& 0 \leq X \leq 0.15 \\
& P_{\text {res }}^{+}=P_{\max }(1-X)
\end{aligned}
$$

The deloading factor (X) should be specified in connection with the maximum permitted limit of the DFIG rotor speed. Aerodynamics, pitch angle controller, servo system, the MPP loaded mechanism and a secondary frequency ribbon described in Figure 6 are part of the frequency control programme for the loaded wind turbine. Fig. 6 displays an ANN controller as opposed to a fixed decreases in the suggested frequency control. This controller provides fast injection capacity to briefly improve frequency behaviour.

The system frequency characteristics of all unknown parameters include maximum inclination and time setup. In order to obtain the expected frequency behaviour, the proper number of parameters must be determined. The purpose in this section is thus to discover an adequate combination of unknown features to optimise the frequency of the microgrid.

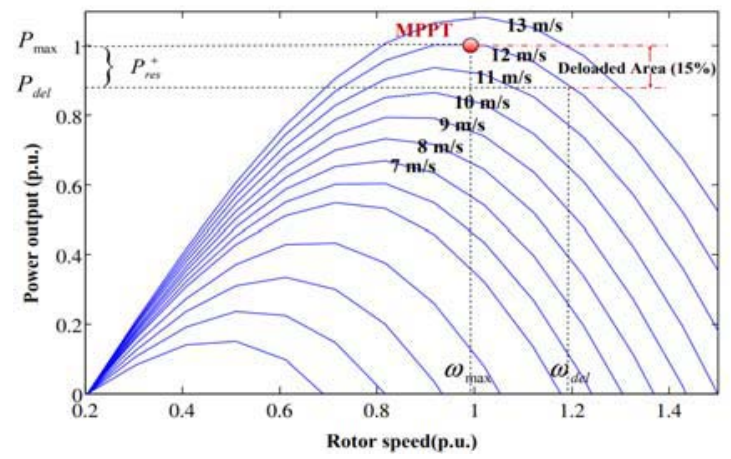

Fig. 5. MPPT and deloaded power curve wind turbine generator.

\section{The Artificial neural network}

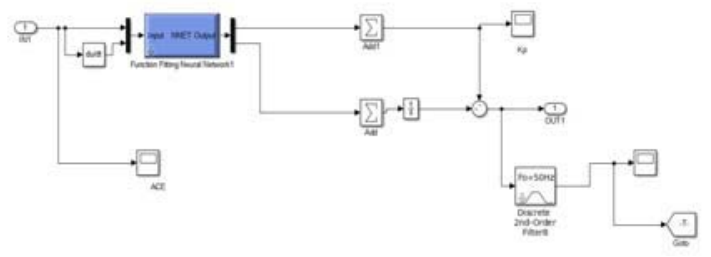

Fig. 6. The wind turbine generator system is proposed with an ANN controller. 
A controller (ANN) that regulates the inputs of each area in the power system collectively will be examined. As a backpacking algorithm, the neural network rule is utilized. The dynamic power system is distributed across time and [SI] is used for the dynamic nature of the behaviour. Figure 2 shows the system $j$ and the ANN controller. Instead of a power system, no emulator is required. The permanent time power system is sampled at 0,01 seconds throughout this techniques, while the dynamic system performance is achieved in a discrete time field by means of the resolution of the State Space Equations. In the system equations of this scenario no $\mathrm{AP}$, fi $(\mathrm{i}=1, \ldots 3)$, state variables are available, but for each area there are five State variables available. ANN controller output is also included in the power system inputs. A three-layer percepton is a 20-block neural network controller. The second cloaked layer consists of 10 neurons and the first cloaks of 3 neurons. The ANN inputs comprise 15 inputs of state and 3 inputs ( $\mathrm{i}=1$, etc.) and are controlled by three outputs from the electric inputs of each zone. The unipolar image sigmoid function is a function of activation. The rear spreading rule shows how the neural network's weight has been changed to minimise the features $\mathrm{E}=1 / 2 \mathrm{e}^{\wedge} \mathrm{T}$ e Weight Transition. This imitates the transition from synaptic to the process of learning the human brain. The back spread rule modifies weights via optimising descent gradients. The weights fluctuate with gradient descent optimization due to the reproduction of the backdrop.

$$
A w_{i j}^{1}=-i \frac{\partial E}{\partial w_{i j}^{1}}
$$

where In the following stratum the neuron I binding with neuron $\mathrm{j}$ is the weight. For the research, two layers, a hidden layer and an output layer consist of the neural network controller. Therefore, there's one, two or two. The positive true figure is the pace of education that is identical to the steps in gradient optimisation. The error gradient on the output layer can be calculated easily:

$$
a_{j}^{3}=-e_{j} f_{j}^{3}
$$

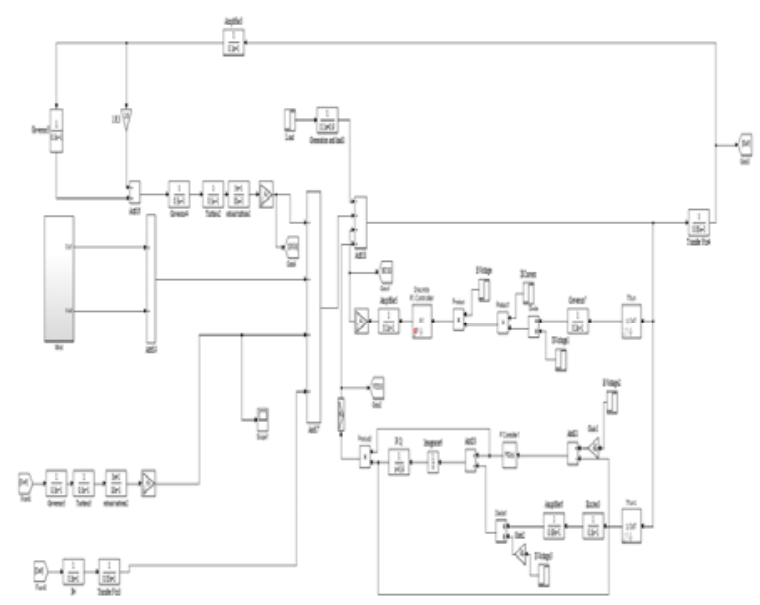

Fig.7. FESS, WTG, DEG, FC, BESS simulation micro-grid diagram.

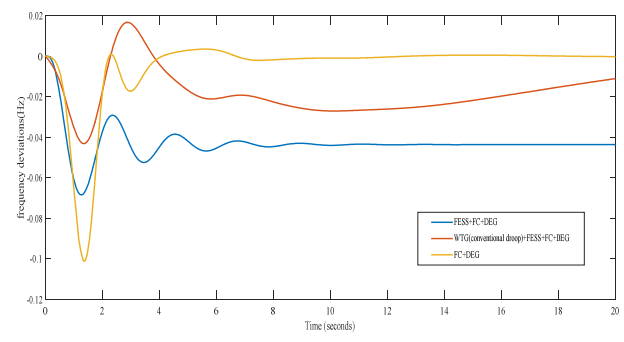

Fig. 8. Frequency deviation curve for different energy management strategies (DERs).

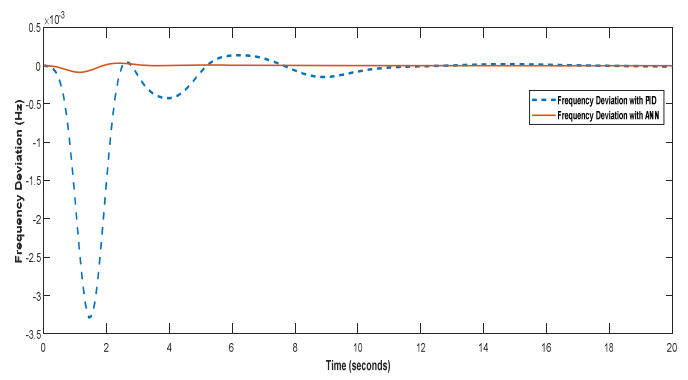

Fig.9. Comparison of ANN and PID controller frequency deviations

It is shown in Figure 10 that differences in the links between sections of the power system are given for the usage of both ANN controllers against load disturbance.

\section{Use of ANN Controller for wind power fluctuations}

It should be the micro grid's constant load. However, due to a load disturbance in any section of the system in question, meteorological changes, in particular dashed line, show the performance of the ANN controller. Data indicate that in the initial area of the power system the ANN controller is superior with an increasing step load to the conventional integrated controller.

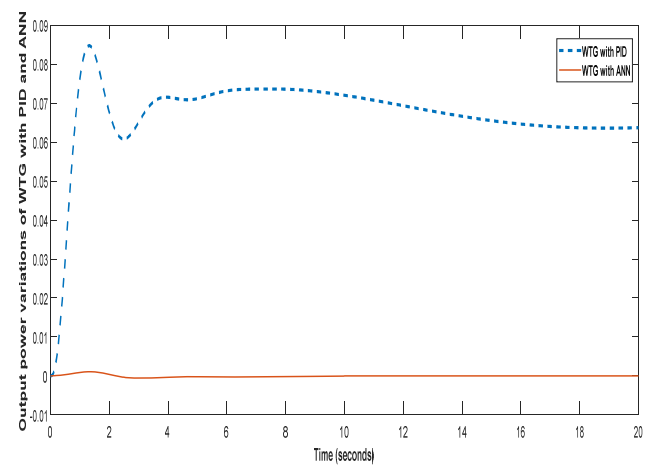

Fig. 10. Power divergence for the ANN and PID controller wind turbine generator. 
Wind speeds contributed to wind power changes throughout this era. The variation in wind speed is the same as the curve shown in Fig. 18. Results showed in Fig. 19 that the suggested ANN technique successfully restricts, prevents and exceeds traditional damping the severe impacts of wind fluctuations.

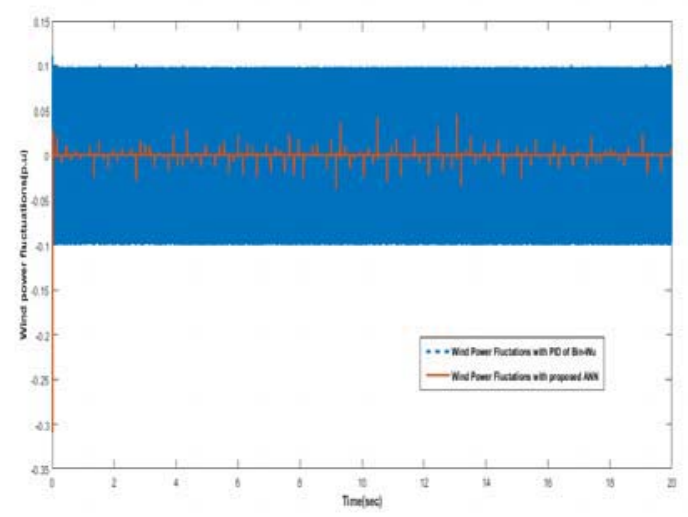

Fig. 11. ANN and PID controller power flucations of WTG.

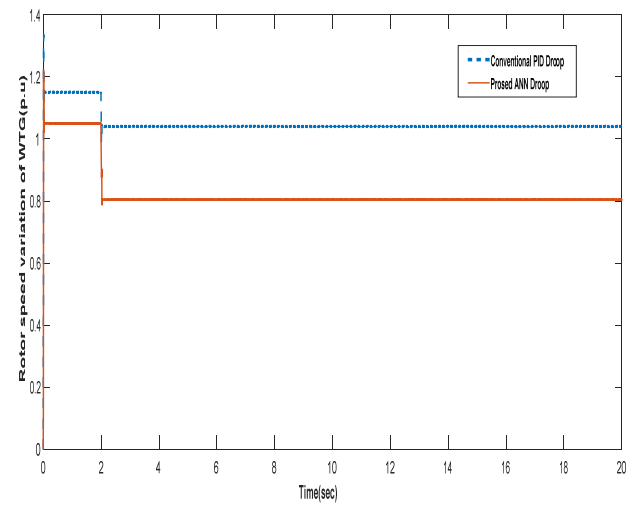

Fig. 12. Variance of the rotor speed of WTG in ANN and PID.

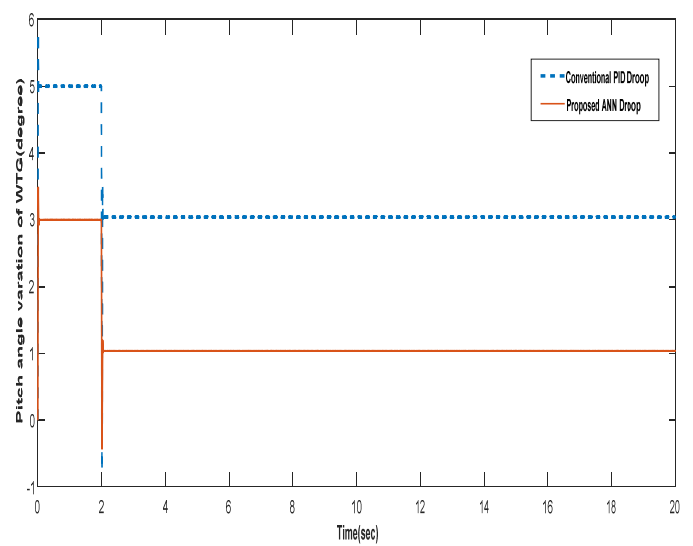

Fig. 13. Change of the WTG pitch angle of the ANN and PID controller.
Table 1. Comparaison of different parameters with PID and ANN controller

\begin{tabular}{|c|c|c|}
\hline S.no. & PID & ANN \\
\hline $\begin{array}{c}\text { Frequency } \\
\text { deviations }\end{array}$ & $\begin{array}{c}\mathrm{T}_{\mathrm{s}}=11 \mathrm{sec} \\
\text { Undershoot }=1.8 \mathrm{sec}\end{array}$ & $\begin{array}{c}\mathrm{T}_{\mathrm{s}}=10 \mathrm{sec} \\
\text { Undershoot }=1 \mathrm{sec}\end{array}$ \\
\hline Power deviations & $\mathrm{T}_{\mathrm{s}}=0.62 \mathrm{sec}$ & $\mathrm{T}_{\mathrm{s}}=0 \mathrm{sec}$ \\
\hline Power fluctuations & +0.1 to- 0.1 & +0.04 to -0.04 \\
\hline Rotor speed & $\mathrm{T}_{\mathrm{s}}=1.01 \mathrm{p} . \mathrm{u}$ & $\mathrm{T}_{\mathrm{s}}=0.8 \mathrm{p} . \mathrm{u}$ \\
\hline Pitch angle & $\mathrm{T}_{\mathrm{s}}=3^{0}$ & $\mathrm{~T}_{\mathrm{s}}=1^{0 .}$ \\
\hline
\end{tabular}

Wind turbine generator variations in power fluctuations has been reduced to zero with these controller. Rotor speed and pitch angle improved of its performance. Finally the change in frequency with in permissible limits are achieved with the help of ANN controller. Tha analysis is shown in Table 1.

\section{Conclusion}

This study is used with ANN for the autonomous control of electricity generation. The transient behaviour of each region frequency and the linear fluctuations of power of the WTG power system are taken into account in all areas of load disturbance. For example, FESS participation in the LFC system through high density energy storage and power exchange with generators of the microgrid. The usual drop in the dynamic WTG model cannot solve the isolated micro grid complexity. The electricity system is mimicked by various source units like hydro and thermal power systems. An additional degree of freedom that increases distortion of the loop system, removing the effects of more interruption poles. These equations are directly used by the integrated controller and the proposed ANN controller in non-linear state space energy supply equations during power regulation. The results of the simulation show that the ANN controller works well against load disturbances in each power system area than a conventional integrated controller.

\section{References}

1. Khalghani MR, Khooban MH, Moghaddam ME, Vafamand N, Goodarzi M., Int J Electr Power Energy Syst ,75,311 (2016)

2. Hosseinzadeh M, Salmasi RF, IEEE Trans Sustain Energy ,6,675 (2015)

3. Lopes PJ, Moreira CL, Mdureria AG, IEEE Trans Power Syst ,21,916 (2006)

4. Olivares DE, Sani M-A, Etemadi AH,IEEE Trans on Smart Grid ,5,1905 (2015)

5. Dhillon SS, Lather JS, Marwaha S, Int J Electr Power Energy Syst ,79,196 (2016)

6. Hossein MJ, Pota HR, Mahmud MA, Aldeen M,IEEE Trans Sustain Energy,6 , 1067 (2009)

7. Souza Ribeiro, LA, De Lima, De Matos JG, Savedra OR, IEEE Trans,2 ,1 (2011)

8. Ataee S. Bevrani MR, Feizi, IEEE Trans. Smart Grid ,7, 706 (2006) 
9. Kim SK, Kim JM and Jeon JH, IEEE International Symposium, Aalborg, Denmark, June Power Electronics for Distributed Generations Systems (2012)

10. Mojica. Mojica, Nava E, Macana CA, Quijano N. IEEE Power Systems ,44, 306(2014)

11. Wu B, Lang Y, Zargari N, Kouro S ,WielyIEEE Press; New York (2011)

12. Yang J, Zhili Z, Yufei $T$, Jun $Y$, Habio $H$, YunliangW,8(3):2145-64energy sources(2015)

13. Zheng Sh, Tang T, Lu Sh, Ye B, Trans ISA, 54 ,539 (2013)

14. Engine Yesil. Engine Yesil, 15:00-12: Appl Soft Compartment (2014)

15. Mahalle,G.,Salunke,O,Kotkunde,N.,Gupta,A.K. ,Singh, S.K., Journal of Materials Research and Technology, 8, 2130 (2019)

16. Khooban MH, Niknam T, Blaabjerg F, Davari P,ISA Trans, 65, 20 (2016)

17. Ramtharan G, Ekanayake JB, Jenkins N. IET Renew Power Gener 1,3 (2007)

18. De Almeria RG, D-Castronuovo E, P Lopes JA. IEEE Trans Power Syst ,2,718 (2006)

19. De Almeida RG, P Lopes JA. IEEE Trans Power Syst ,22, 944 (2007)

20. Moutis P, Loukarakis E, Papathanasiou S, et al. IEEE Bucharest Power Tech ,1(2009)

21. Maurici JM, Marano A, G-Exposito A, Martinez Ramos JL, IEEE Trans on Power Syst 24,173(2008)

22. Abazari A, Ghazavi Dozein M, Monsef H, J Franklin Inst ,355, 6262 (2018)

23. Ghafouri A, Milimonfared J, B Gharehpetian G, IEEE Syst J ,99(1):1-9(2017)

24. Vidyanandan KV, Senroy N,IEEE Trans Power Syst ,28, 839 (2013)

25. Zhang W, Fang K, IET Gener Transm Distrib 11,1 (2017)

26. Sridevi, J., Rani, V.U., IEEE ICECIE Proceedings , (2019)

27. Sridevi, J., Amarnath, J., Govinda Rao, G., International Review on Modelling and Simulations , 4, 2485 (2011)

28. Sridevi, J., Amarnath, J., Govinda Rao, G., International Review on Modelling and Simulations , 4,1799 (2011)

29. Dhanlaxmi,B.,ApparaoNaidu,G.,Anuradha,K, Procedia Comp Sci, 46, 432 (2015) 